\title{
Social Trajectory and Body Image Satisfaction in Childbearing Women
}

\author{
Ana Henriques • Luís Alves • Elisabete Alves • \\ Susana Silva $\cdot$ Henrique Barros $\cdot$ Ana Azevedo
}

Published online: 31 October 2014

(C) Springer Science+Business Media New York 2014

\begin{abstract}
Advantaged socioeconomic position (SEP) is associated with lower body image satisfaction (BIS) among women. However, motherhood and social trajectory (an individual's path from childhood SEP to adulthood SEP) could change this relationship. We aimed to assess the association between social trajectory and BIS immediately before getting pregnant in primiparous and multiparous mothers of a birth cohort. The birth cohort Generation XXI was assembled after delivery, in Porto, in 2005-2006. This analysis includes 5,470 women. Women's and their parents' education were used as indicators of adulthood and childhood SEP, respectively. Social trajectory was
\end{abstract}

Electronic supplementary material The online version of this article (doi:10.1007/s10995-014-1629-2) contains supplementary material, which is available to authorized users.

\footnotetext{
A. Henriques $(\varangle) \cdot$ L. Alves $\cdot$ E. Alves $\cdot$ S. Silva $\cdot$ H. Barros $~$ A. Azevedo

EPIUnit - Institute of Public Health, University of Porto, Porto, Portugal

e-mail: alhenriques@med.up.pt

L. Alves

e-mail: luisaaalves@gmail.com

E. Alves

e-mail: ealves@med.up.pt

S. Silva

e-mail: susilva@med.up.pt

H. Barros

e-mail: hbarros@med.up.pt

A. Azevedo

e-mail: anazev@med.up.pt

L. Alves · E. Alves - H. Barros · A. Azevedo

Department of Clinical Epidemiology, Predictive Medicine and

Public Health, University of Porto Medical School, Alameda

Prof. Hernâni Monteiro, 4200-319 Porto, Portugal
}

classified as stable-high, upward, stable-low, downward, according to both education variables. BIS was assessed with Stunkard silhouettes immediately after birth as the difference between perceived body size before the index pregnancy and ideal body size. Odds ratios (OR) between social trajectory and BIS were computed using multinomial logistic regression (women satisfied with body image were the outcome reference category), adjusting for age and prepregnancy body mass index. In primiparous women, no association was found between childhood SEP, adulthood SEP or social trajectory and feeling too small or too large regarding their ideal figure. Multiparous women with a downward social trajectory presented a higher likelihood of dissatisfaction [too small: adjusted OR 2.21, $95 \%$ confidence interval (95\% CI) 1.10-4.46; too large: adjusted OR 1.64, $95 \%$ CI 1.07-2.51]. Downward social trajectory was associated with a higher likelihood of dissatisfaction with body image in multiparae, while there was no effect among primiparae.

Keywords Social trajectories - Body image satisfaction . Motherhood · Parity

\section{Introduction}

Body image is a multidimensional construct that relates to a person's perceptions, feelings and thoughts about his or her body and contains both cognitive (attitudinal) and affective dimensions. One of the components of the attitudinal dimension is the evaluative component represented by the discrepancy between self and ideal perceived images to measure body satisfaction $[1,2]$. Although body image is a complex construct, the major research focus has been on body's appearance, mainly in high income settings 
across the world, where a female beauty ideal emphasizes the desirability of thinness [3, 4].

Socioeconomic position (SEP) is an aggregate term that includes resource-based (income, wealth, educational credentials) and prestige-based measures (ranked in a social hierarchy evaluated with reference to people's access to goods, services and knowledge), linked to both childhood and adulthood social class [5]. Most often, only some of these indicators are used and previous studies have shown that education is the component of adult SEP that contributes most to body dissatisfaction [6].

In Western societies, women from a higher SEP are thinner than women from lower SEP [7], illustrating how social conditions are written into the bodies, producing population patterns of health [8]. Part of the protection against weight gain in this group could result from a higher frequency of weight monitoring, a lower threshold for defining themselves as overweight, and a greater likelihood of deliberate efforts at weight control [9].

Several studies have demonstrated that, for a given body size, women from a higher SEP are more often dissatisfied with their body than women from a lower SEP $[9,10]$. However, these findings have been based on women's SEP in adulthood, and little attention has been paid to the household SEP during her childhood or to intergenerational social trajectory taking into account a life course perspective. Social trajectory is a lifelong evolution of the volume and composition of an individual's capital (social, cultural, economic and/or symbolic), combined with his/ her parents' asset volume and structure and can be described as upward, downward or stationary [11]. Changes in social circumstances, or intergenerational movement between social classes, might entail a transition in terms of priorities and resources related to weight and appearance, or a shift in experience of social norms regarding the appeal of particular body types [6], particularly when considered in the context of women who have recently given birth.

The experience of pregnancy, with marked physical and emotional changes, raises questions in a woman about her body image perception [12]. The weight and shape changes related to pregnancy can be distressing for some women but neutral or even liberating for others [13]. Moreover, there is a substantial discrepancy between the expectations and the reality of motherhood specially for primiparous women, for whom motherhood is associated with much uncertainty, leaving them more prone to a romanticized vision of this new role [14]. Body image satisfaction (BIS) influences maternal body weight after delivery [15], making it relevant to understand the determinants of BIS in specific subgroups of childbearing women.

Within this framework, the aim of this study was to assess the relation of childhood SEP, adulthood SEP and social trajectory with BIS immediately before pregnancy in primiparous and multiparous mothers of a Portuguese birth cohort.

\section{Methods}

\section{Study Design}

This study is based on the birth cohort Generation XXI which has been previously described [16]. Briefly, 8,495 mothers, who gave birth to 8,647 infants, were recruited in 2005-2006 at all five public maternity units covering six municipalities of the metropolitan area of Porto, Portugal. All mothers residing in the catchment area who delivered a live-born child (gestational age $\geq 24$ weeks) in one of the five units were eligible and subsequently invited to join the study, 24-72 h after delivery. At birth, $91.4 \%$ of the invited mothers accepted to participate.

For the current analysis and since body image perception is expected to change during pregnancy, we excluded 313 participants who had been recruited during the first trimester of pregnancy to address specific objectives [17]. Women with missing data on potentially confounding variables of the relationship between social trajectory and BIS were excluded from analysis. Thus, 2,712 women without data on education, household monthly income, working condition, highest parents' education, having a maid, going away on vacations, car ownership, home ownership, body mass index (BMI), or BIS were excluded. The present study is based on 5,470 women who were recruited and evaluated during their hospital stay for childbirth and had complete information regarding the key variables considered.

Compared to excluded women, those included in the analyses were younger mean [standard deviation (SD)]: 29.0 (5.6) vs. 30.4 (5.4) years, independent sample $t$ test: $p<0.001\}$, had a lower household income ( $>1,500$ euros/ month: $26.8 \%$ vs. $29.5 \%$, Chi-square test: $p=0.049$ ), less frequently had a car, maid or went away on vacations when they were 12 years old (all amenities: 8.4 vs. $9.8 \%$, Chi-square test: $p=0.024$ ) and were more often unemployed (21.1 vs. $17.9 \%$, Chi-square test: $p=0.001$ ), despite the negligible magnitude of differences. However, included and excluded women did not differ in relation to the main variables such as a women's education, highest parents' education, parity, BMI, BIS or social trajectory.

\section{Data Collection and Variables Definition}

Trained inquirers collected data on demographic, socioeconomic, lifestyles, obstetric history, anthropometrics and body image (self and ideal) characteristics, in face-to-face 
interviews using standardized questionnaires. Data collection took place 24-72 $\mathrm{h}$ after delivery, during the hospital stay.

The outcome variable, BIS before pregnancy, was calculated as the difference between perceived self body size and ideal body size, both assessed after delivery, by the Stunkard silhouettes [18]. This scale consists of nine numbered silhouette figures that increase gradually in size from very thin to very obese (1-9, respectively). Self body size was the number of the figure selected by participants in response to "choose the figure that reflects how you think you looked before you got pregnant" and ideal body size in response to "choose your ideal figure". BIS was categorized as being satisfied with one's body image (difference between self and ideal body size $=0$ ); feeling too small regarding one's ideal (difference between self and ideal $<0$ ); and feeling too large regarding one's ideal (difference between self and ideal $>0$ ).

We studied multiple variables as potential predictors of women's BIS. Women reported their age at the time of delivery, and this was later categorized it into four ordinal categories: $<25,25-29,30-34$ and $\geq 35$ years. Parity was recorded as the number of deliveries, including the index one and was afterwards categorized into having only one delivery (primiparous) and more than one (multiparous). Women were inquired about their prepregnancy weight to the nearest $0.1 \mathrm{~kg}$. Immediately after birth, height was measured (without shoes) by the inquirers to the nearest $0.1 \mathrm{~cm}$ and, when measurement was not possible, height was reported by the mother as registered in the identity card. Mother's prepregnancy BMI was calculated and later categorized into four ordinal categories according to the standard World Health Organization definition: underweight $\left(<18.5 \mathrm{~kg} / \mathrm{m}^{2}\right)$, normal $\left(18.5-24.9 \mathrm{~kg} / \mathrm{m}^{2}\right)$, overweight $(25.0$ $\left.29.9 \mathrm{~kg} / \mathrm{m}^{2}\right)$ and obese $\left(\geq 30 \mathrm{~kg} / \mathrm{m}^{2}\right)$ [19]. Women's and their parents' education were self-reported by the delivering women as the total number of years of formal education completed and subsequently converted into ordinal categories (women's: $\geq 12$, $10-11,5-9$, $\leq 4$; their parents: $\geq 12,9-11,6-8,4-5,<4)$. Household monthly income was inquired using previously defined categories (less than $500 €, 500-1,000 €, 1,001-1,500$ $€$, more than $1,500 €)$. Working condition was defined as employed, unemployed, housewife and others (student or retired). Amenities including having a car, maid and/or going away on vacations were categorized according to the number of possessions these women had when they were 12 years of age.

Concerning the main exposure variables, and since education is often used as a generic measure of SEP [20], adulthood SEP was defined using mother's education immediately after delivery. Childhood SEP was defined according to the highest parents' education when these women were 12 years old. Thus, we categorized women's education and their parents' education into four and five categories, respectively, to explore the graded shape and the location of any thresholds in the association between education and BIS (supplementary file, available online). After that, and based on the interpretation of those associations, the researchers decided which were the best cutoff points for both variables in order to use only two classes (high and low) for the definition of social trajectory from childhood to adulthood. Then, parents' education was considered high if at least one of the parents had 6 or more years of education and women's education was high when they had more than 9 years of education. To examine the effect of social trajectory on BIS, a variable comprising the four different combinations of childhood and adulthood SEP categories was defined: stable-high: parents' education $\geq 6$ and woman's education $>9$ years; upward trajectory: parents' education $<6$ and woman's education $>9$ years; stable-low: parents' education $<6$ and woman's education $\leq 9$ years and downward trajectory; parents' education $\geq 6$ and woman's education $\leq 9$ years.

\section{Statistics}

Statistical analyses were performed using Stata 11.0 (College Station, TX, 2009). Sample characteristics were presented as counts and proportions for all categorical variables and mean and standard deviation (SD) for normally distributed continuous variables. Proportions were compared using the Chi-square test and continuous variables with independent-sample $t$ test. Odds ratios (OR) with the respective $95 \%$ confidence intervals (95\% CI) for the association between childhood SEP, adulthood SEP and social trajectory with BIS were computed using multinomial logistic regression models, taking women satisfied with their body image as the reference category of the outcome. Multinomial logistic regression was used due to the existence of more than two categories in the dependent variable.

Separate models were built for primiparous and multiparous women because we found different effects of social trajectories on BIS according to parity ( $p$ for the interaction in the age and BMI-adjusted model considering the too large extreme: 0.012). For each group, two final models were fitted: one adjusting only for age and another adjusting additionally for BMI before pregnancy. From a list of a priori potential confounders, these two were the only variables significantly associated with BIS $(p<0.05)$ and confounding the main association (assessed by the change of the association between social trajectory and BIS by at least $10 \%$ ).

\section{Ethics}

The study protocol was approved by the Ethics Committee of Hospital de São João. All participants received an explanation of the purposes and design of the study and gave written informed consent for the evaluation. 
Table 1 Participants' characteristics, according to parity $(\mathrm{n}=5,470)$

\begin{tabular}{|c|c|c|c|}
\hline & \multicolumn{3}{|l|}{ Parity } \\
\hline & $\begin{array}{l}\text { Primiparae } \\
\mathrm{n}(\%)\end{array}$ & $\begin{array}{l}\text { Multiparae } \\
\mathrm{n}(\%)\end{array}$ & $p$ \\
\hline Overall & $3,166(57.9)$ & $2,304(42.1)$ & \\
\hline \multicolumn{4}{|l|}{ Age (years) } \\
\hline$<25$ & $992(31.3)$ & $292(12.7)$ & \\
\hline $25-29$ & $1,283(40.5)$ & $627(27.2)$ & \\
\hline $30-34$ & $659(20.8)$ & $739(32.1)$ & \\
\hline$\geq 35$ & $232(7.3)$ & $646(28.0)$ & $<0.001$ \\
\hline \multicolumn{4}{|l|}{ BMI before pregnancy $\left(\mathrm{Kg} / \mathrm{m}^{2}\right)$} \\
\hline$<18.5$ & $162(5.1)$ & $69(2.9)$ & \\
\hline $18.5-24.9$ & $2,206(69.7)$ & $1,352(58.7)$ & \\
\hline $25.0-29.9$ & $585(18.5)$ & $605(26.3)$ & \\
\hline$\geq 30$ & $213(6.7)$ & $278(12.1)$ & $<0.001$ \\
\hline \multicolumn{4}{|l|}{ Body image satisfaction } \\
\hline Too small & $317(10.0)$ & $179(7.8)$ & \\
\hline Satisfied & $1,521(48.0)$ & $918(39.8)$ & \\
\hline Too large & $1,328(42.0)$ & $1,207(52.4)$ & $<0.001$ \\
\hline \multicolumn{4}{|l|}{ Childhood SEP } \\
\hline \multicolumn{4}{|l|}{ Highest parents' education (years) } \\
\hline$\geq 12$ & $446(14.1)$ & $247(10.7)$ & \\
\hline $9-11$ & $305(9.6)$ & $143(6.2)$ & \\
\hline $6-8$ & $379(12.0)$ & $181(7.9)$ & \\
\hline $4-5$ & $1,786(56.4)$ & $1,433(62.2)$ & \\
\hline$<4$ & $83(2.6)$ & $155(6.7)$ & \\
\hline Does not know & $167(5.3)$ & $145(6.2)$ & $<0.001$ \\
\hline \multicolumn{4}{|l|}{ Amenities (car, maid, vacations) } \\
\hline All & $291(9.2)$ & $171(7.4)$ & \\
\hline 2 & $803(25.4)$ & $439(19.0)$ & \\
\hline 1 & $1,081(34.1)$ & $602(26.1)$ & \\
\hline None & $991(31.3)$ & $1,092(47.4)$ & $<0.001$ \\
\hline \multicolumn{4}{|l|}{ Adulthood SEP } \\
\hline \multicolumn{4}{|l|}{ Education (years) } \\
\hline$\geq 12$ & $1,547(48.9)$ & $746(32.4)$ & \\
\hline $10-11$ & $286(9.0)$ & $154(6.7)$ & \\
\hline $5-9$ & $1,246(39.4)$ & $1,102(47.9)$ & \\
\hline$\leq 4$ & $87(2.8)$ & $302(13.1)$ & $<0.001$ \\
\hline \multicolumn{4}{|l|}{ Household monthly income $(€)$} \\
\hline$>1,500$ & $856(27.0)$ & $612(26.6)$ & \\
\hline $1,001-1,500$ & $855(27.0)$ & $541(23.5)$ & \\
\hline $500-1,000$ & $931(29.4)$ & $745(32.3)$ & \\
\hline$<500$ & $179(5.6)$ & $185(8.0)$ & \\
\hline Does not know/prefers not to answer & $345(10.9)$ & $221(9.6)$ & $<0.001$ \\
\hline \multicolumn{4}{|l|}{ Working condition } \\
\hline Employed & $2,306(72.8)$ & $1,533(66.5)$ & \\
\hline Unemployed & $626(19.8)$ & $527(22.9)$ & \\
\hline Housewife & $89(2.8)$ & $223(9.7)$ & \\
\hline Others & $145(4.5)$ & $21(0.9)$ & $<0.001$ \\
\hline
\end{tabular}


Table 1 continued

\section{Parity}

\begin{tabular}{lll}
\hline $\begin{array}{l}\text { Primiparae } \\
\mathrm{n}(\%)\end{array}$ & $\begin{array}{l}\text { Multiparae } \\
\mathrm{n}(\%)\end{array}$ & $p$
\end{tabular}

Intergenerational SEP

Social trajectory (parents' and women's education)*

Stable-high

Upward trajectory

Stable-low

Downward trajectory

Comparisons between primiparae and multiparae were made using the Chi-square test

$B M I$ body mass index, SEP socioeconomic position

$* \mathrm{n}=5,158$ due to the exclusion of women who did not know their parents' education

Fig. 1 Age-adjusted and ageand body mass index-adjusted odds ratio for the association between highest parents' education, woman's education and social trajectory with body image satisfaction, estimated by multinomial logistic regression, among primiparous and multiparous women
PRIMIPARAE

MULTIPARAE

Age-adjusted Age-and BMI adjusted Age-adjusted Age-and BMI adjusted
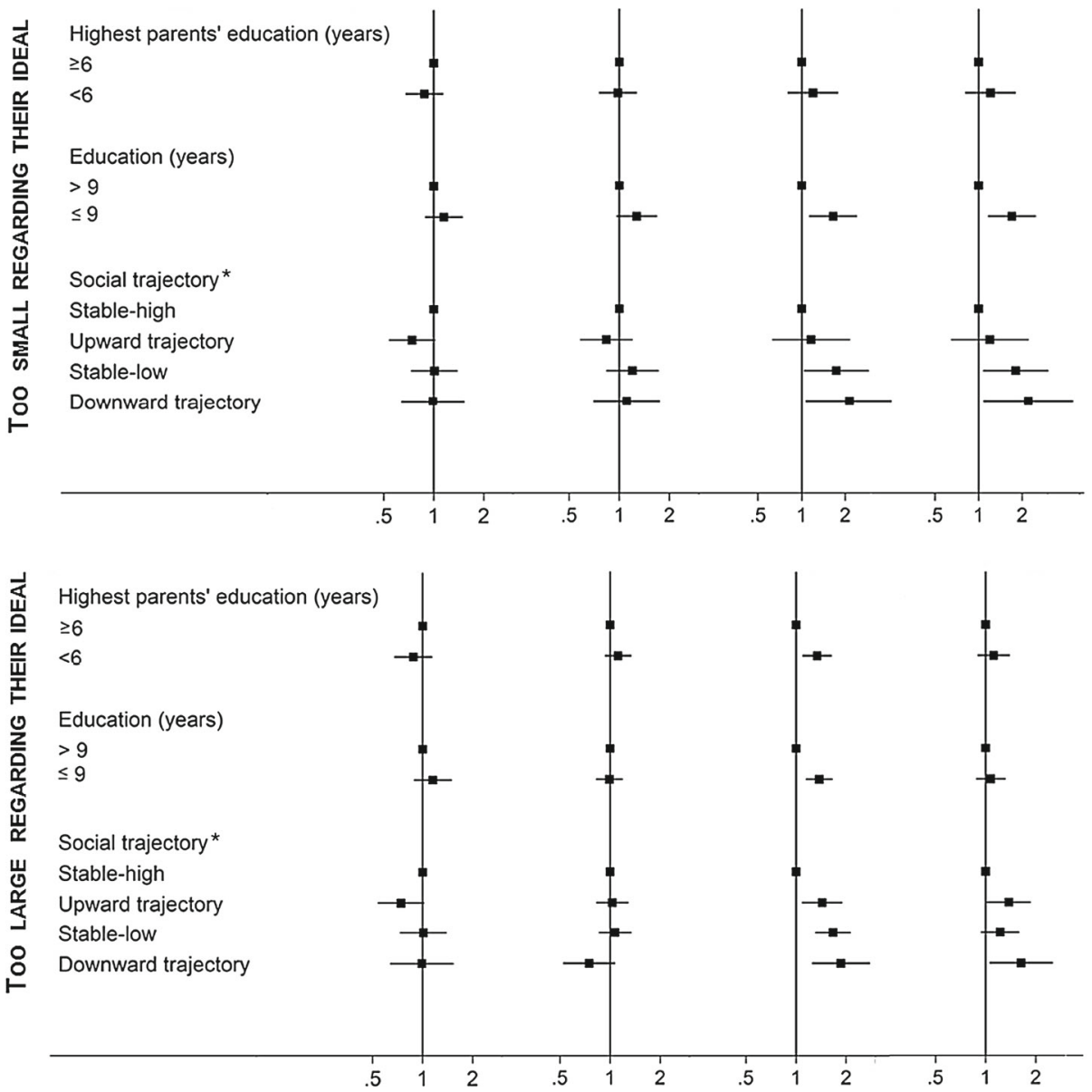

*Stable-high: parents' education $\geq 6$ and woman's education $>9$ years; upward trajectory: parents' education $<6$ and woman's education $>9$ years; stable-low: parents' education $<6$ and woman's education $\leq 9$ years and downward trajectory; parents' education $\geq 6$ and woman's education $<9$ years 


\section{Results}

In this sample of Portuguese women, 57.9 and $42.1 \%$ were primiparous and multiparous, respectively. Also, $7.3 \%$ of primiparae and $28.0 \%$ of multiparae were at least 35 years old and the prevalence of obesity was higher among multiparous women (12.1 vs. $6.7 \%$ ). Concerning BIS, more than half of the multiparous women reported a self body image larger than their ideal and this prevalence was lower among primiparous women (52.4 vs. $42.0 \%$ ).

With regards to women's childhood, both parents of $59.0 \%$ of primiparae had less than 6 years of education, while this percentage was considerably higher in multiparae $(68.9 \%)$. Almost one-third of primiparae and half of the multiparae possessed none of the 3 types of amenities considered. Regarding their own education, almost six out of ten primiparous women had more than 9 years of education while less than four out of ten multiparous women had the same degree. In both primiparae and multiparae, $27 \%$ had a household monthly income above 1,500 euros and multiparous were slightly more frequently unemployed (22.9 vs. $19.8 \%$ ). Almost a third of primiparae were in the stable-low category of social trajectory compared to more than half of multiparae in this same class (Table 1).

The detailed analysis on the association of socioeconomic characteristics concerning women's childhood and adulthood with BIS is presented in the supplementary file, available online. In general, when compared to the reference category for each variable, socioeconomically disadvantaged women were more likely to be dissatisfied with body image. The associations were stronger among multiparae and the effects on feeling too large regarding one's ideal body were attenuated by the adjustment for BMI.

Figure 1 depicts the association of childhood SEP, adulthood SEP, and social trajectory with BIS among primiparous and multiparous women. In both groups and after adjusting for age and BMI, no significant association was found between highest parents' education and feeling too small in both groups of women (primiparous: age-and BMI- adjusted OR 0.98, $95 \%$ CI 0.76-1.28; multiparous: age-and BMI- adjusted OR 1.21, 95 \% CI 0.82-1.79).

Likewise, there were no differences in feeling too large between women whose parents had less than 6 years of education and 6 or more (primiparous: age-and BMIadjusted OR 1.12, $95 \%$ CI 0.94-1.32; multiparous: age-and BMI- adjusted OR 1.11, $95 \%$ CI 0.90-1.39). A significant association was found between women with 9 or less years of education and feeling too small, among multiparae (age-and BMI-adjusted OR 1.70, $95 \%$ CI 1.16-2.48).

The association of mother's adulthood SEP with dissatisfaction towards the "too large extreme" was explained by BMI before pregnancy in primiparous and multiparous women (age-and BMI-adjusted OR 0.99, $95 \%$ CI
0.82-1.18 and age-and BMI-adjusted OR 1.07, $95 \% \mathrm{CI}$ $0.87-1.31$, respectively).

Overall, for women with only one delivery, no effect of social trajectory was observed towards both extremes of dissatisfaction, after taking age and BMI into account. In multiparous women, the likelihood of feeling too small, independently of age and BMI, increased progressively across categories of social trajectory up to an age-and BMIadjusted OR of 2.21, $95 \%$ CI 1.10-4.46 in the downward trajectory class. Regarding the too large extreme of body image dissatisfaction, though women's and parents' education did not have a considerable impact per se, the odds of feeling too large increased across the categories of social trajectory up to an age-and BMI-adjusted OR of 1.64, $95 \%$ CI 1.07-2.51 in the downward trajectory class (Fig. 1).

\section{Discussion}

We examined the relation of childhood SEP, adulthood SEP and social trajectory with BIS before pregnancy in mothers of a Portuguese birth cohort. Among primiparous women, there was no association between childhood SEP, adulthood SEP or social trajectory and being dissatisfied with body image. In this group of women, BMI was the main determinant of feeling too small or too large, with no additional effect of SEP or social trajectory. In multiparous women, patterns of social trajectory had differential effects on the discrepancy between perceived and ideal body size, and those who experienced a downward social trajectory from childhood to adulthood had the highest probability of being dissatisfied with their appearance in either direction.

Several studies indicate that socioeconomically advantaged women are more often dissatisfied with their bodies than socioeconomically disadvantaged women $[10,21]$ probably due to a higher pressure for thinness induced by social norms that is commonplace in high-income regions [4]. A cohort of middle-aged British women showed that those who experienced a downward trajectory were more satisfied with their appearance than stable non-manual women, independently of their BMI [6]. Our results in women who recently delivered a child point to an opposite direction, probably due to the recent experience of motherhood that can change how women perceive their appearance and their physical ideals.

It is well established that childbearing is associated with permanent weight gain in women [22] and the reversibility of weight gain is known to be a concern [23], possibly anticipating dissatisfaction with body image [24]. To eliminate the possible effect of weight gain on BIS due to previous pregnancies, we performed a stratified analysis by parity. Since clearly distinct associations were observed by parity, the results were presented separately for primiparous and multiparous. This dissatisfaction may be less apparent in 
primiparae, attenuated by the enchantment of motherhood and all uncertainty regarding the unknown role of mother and the body changes to be expected. Thus, women may view the body changes as transient and unique, being able to assimilate them without distress independently of their SEP. In women who had been pregnant before, BIS may decline due to previous experience, such as the difficulty in returning to their prepregnancy weight and this decline may be larger in women presenting a downward social trajectory.

Women who experienced a downward trajectory were the ones who felt more frequently dissatisfied, not only by feeling too large but also too small, and the association was stronger than the association between adulthood SEP and BIS. This pattern is highly suggestive of how social conditions become embodied and generate dissatisfaction with body image [25], particularly when these are worse than in earlier stages of life [26].

To help interpret the results, they should be framed within the socioeconomic and cultural context that shapes the access to education in Portugal in the last three decades. Our sample encompasses a generation who grew up after important national political and social changes. Women in particular, became more involved in all businesses, professions, schools and universities, culminating in considerable changes involving the role of women in society [27]. In this context, women of this generation who experienced a downward trajectory are a particularly disadvantaged group since their trajectory is opposite to the national trend.

Some limitations of this study should be discussed. The Stunkard silhouettes have some limitations and offer an incomplete view of the body image construct; however this instrument also has some advantages, allowing a uniform approach to study subjects that is not overly time-consuming. The credibility of this tool for scientific research is reinforced by its application in recent investigations [28, 29] and figure drawing scales are still the most widely adopted measures of dissatisfaction with body size [30]. Moreover, this scale is validated in the Caucasian population [31] and only $3 \%$ of this cohort was not European [32]. We are aware that the measurement procedure may determine the significance and strength of the associations between SEP and BIS estimated in a study, which can contribute to the differences between our results and previous literature. Despite the incomplete approach to the body image construct, the meaning of body image satisfaction using a simple measure based on the Stunkard silhouettes is clear and relevant for non-specialists and, from a public health perspective, we believe our estimates validly demonstrate that patterns of social trajectory can have differential effects on the discrepancy between perceived and ideal body size. We used this tool to address BIS before pregnancy. There are no validation studies for the reference to a past time and it would be fruitful to validate the method for this purpose in a future study.
Concerning the social trajectory and SEP measurement, education is not the only variable that measures SEP and, when considering social trajectory, education only covers the social and cultural capital. SEP is a complex attribute and there is not a set of indicators that can describe a person's SEP. Each has advantages and disadvantages [20, 33]. Formal education is nowadays one of the key elements in both the organization of people's daily lives and life courses. Similarly, it is currently one of the axes that are decisive to the unequal distribution of resources and opportunities, conditioning people's social life in different ways and strongly contributing to the structuration of class relationships [27]. We relied on education due to its important established role in health behaviors and outcomes, because it is the most commonly used variable as a generic measure of SEP [20]. Also, previous work showed that education is the component of SEP that contributes most to body dissatisfaction [6]. Approximately $3 \%$ of the women in the cohort were still students at the time of evaluation. However, only $6.8 \%$ of women in the downward trajectory category and $1.7 \%$ of women in the stable-low category of social trajectory could still move across categories with their ongoing education and we believe this is not enough to change the strength of the associations substantially. About the thresholds used to categorize education, our first thought was to define a dichotomous variable comprising having or not completed compulsory education both for mothers and their parents. However, although compulsory education formally changed from 4 to 6 years in 1964 in Portugal, the conditions for the completion of 6 years of compulsory schooling everywhere were only definitively established in the late 1970s. Therefore, for more than 10 years, there was much uncertainty around this subject and it was not possible to define what was compulsory education for each child in different regions over those years. Therefore, since the access to education clearly changed in these last three decades and these two generations had different opportunities to study, it was expected to have different thresholds to define high and low education for women and their parents.

In conclusion, this study provides evidence that social trajectories influences BIS and parity may modify this association: downward social trajectory was associated with a lower BIS in multiparae, while there was no effect among primiparae. This work suggests that social trajectories have different effects on the discrepancy between perceived and ideal body size, likely by influencing both an individual's ideal and the ability to attain it. Taking into account the fact that BIS before pregnancy influences maternal body weight after delivery, a deeper understanding of BIS could help to outline more specific and adequate interventions to women, in order to prevent excessive weight rates in this particular population. 
Acknowledgments The cohort 'Geração XXI' was funded by 'Programa Operacional de Saúde - Saúde XXI, Quadro Comunitário de Apoio III', 'Administração Regional de Saúde Norte' and 'Fundação Calouste Gulbenkian'. Grants from 'Fundação para a Ciência e a Tecnologia' (PIC/IC/83038/2007 and SFRH/BD/72723/2010) are gratefully acknowledged. The authors are also grateful to all of the staff of the Generation XXI team for their contribution to this work.

\section{References}

1. Grogan, S. (2006). Body image and health: Contemporary perspectives. Journal of Health Psychology, 11(4), 523-530.

2. Cash, T. (2002). Cognitive-behavioral perspectives on body image. In T. Cash \& T. Pruzinsky (Eds.), Body image: A handbook of theory, research, and clinical practice (pp. 38-46). New York: Guilford Press.

3. Rysst, M. (2010). "Healthism" and looking good: Body ideals and body practices in Norway. Scandinavian Journal of Public Health, 38(5 Suppl), 71-80.

4. Swami, V., Frederick, D. A., Aavik, T., et al. (2010). The attractive female body weight and female body dissatisfaction in 26 countries across 10 world regions: Results of the international body project I. Personality and Social Psychology Bulletin, 36(3), 309-325.

5. Krieger, N. (2001). A glossary for social epidemiology. Journal of Epidemiology and Community Health, 55(10), 693-700.

6. McLaren, L., \& Kuh, D. (2004). Women's body dissatisfaction, social class, and social mobility. Social Science and Medicine, 58(9), 1575-1584.

7. McLaren, L. (2007). Socioeconomic status and obesity. Epidemiologic Reviews, 29, 29-48.

8. Krieger, N., \& Davey Smith, G. (2004). "Bodies count," and body counts: Social epidemiology and embodying inequality. Epidemiologic Reviews, 26, 92-103.

9. Wardle, J., \& Griffith, J. (2001). Socioeconomic status and weight control practices in British adults. Journal of Epidemiology and Community Health, 55(3), 185-190.

10. Lynch, E., Liu, K., Spring, B., et al. (2007). Association of ethnicity and socioeconomic status with judgments of body size: The Coronary Artery Risk Development in Young Adults (CARDIA) Study. American Journal of Epidemiology, 165(9), 1055-1062.

11. Bourdieu, P. (1984). Distinction: A social critique of the judgment of taste. Cambridge, MA: Harvard University Press.

12. Schmied, V., \& Lupton, D. (2001). The externality of the inside: Body images of pregnancy. Nursing Inquiry, 8(1), 32-40.

13. Tiggemann, M. (2004). Body image across the adult life span: Stability and change. Body Image, 1(1), 29-41.

14. Staneva, A., \& Wittkowski, A. (2013). Exploring beliefs and expectations about motherhood in Bulgarian mothers: A qualitative study. Midwifery, 29(3), 260-267.

15. Henriques, A., Alves, E., Barros, H., et al. (2013). Women's satisfaction with body image before pregnancy and body mass index 4 years after delivery in the mothers of generation XXI. PLoS ONE, 8(7), e70230.

16. Larsen, P. S., Kamper-Jorgensen, M., Adamson, A., et al. (2013). Pregnancy and birth cohort resources in Europe: A large opportunity for aetiological child health research. Paediatric and Perinatal Epidemiology, 27(4), 393-414.

17. Pinto, E., Ramos, E., Severo, M., et al. (2010). Measurement of dietary intake of fatty acids in pregnant women: Comparison of self-reported intakes with adipose tissue levels. Annals of Epidemiology, 20(8), 599-603.

18. Stunkard, A. J., Sorensen, T., \& Schulsinger, F. (1983). Use of the Danish Adoption Register for the study of obesity and thinness. In S. Kety, L. Rowland, R. Sidman, \& S. Matthysse (Eds.), Genetics of neurological and psychiatric disorders (pp. 115-120). New York: Raven.

19. (1998). Clinical guidelines on the identification, evaluation, and treatment of overweight and obesity in adults: executive summary. Expert panel on the identification, evaluation, and treatment of overweight in adults. Am J Clin Nutr, 68(4):899-917.

20. Galobardes, B., Shaw, M., Lawlor, D. A., et al. (2006). Indicators of socioeconomic position (part 1). Journal of Epidemiology and Community Health, 60(1), 7-12.

21. Ogden, J., \& Thomas, D. (1999). The role of familial values in understanding the impact of social class on weight concern. International Journal of Eating Disorders, 25(3), 273-279.

22. Abrams, B., Heggeseth, B., Rehkopf, D., et al. (2013). Parity and body mass index in U.S. women: A prospective 25-year study. Obesity (Silver Spring), 21(8), 1514-1518.

23. Harris, H. E., Ellison, G. T., \& Clement, S. (1999). Relative importance of heritable characteristics and lifestyle in the development of maternal obesity. Journal of Epidemiology and Community Health, 53(2), 66-74.

24. Gavin, A. R., Simon, G. E., \& Ludman, E. J. (2010). The association between obesity, depression, and educational attainment in women: The mediating role of body image dissatisfaction. Journal of Psychosomatic Research, 69(6), 573-581.

25. Krieger, N. (2005). Embodiment: A conceptual glossary for epidemiology. Journal of Epidemiology and Community Health, 59(5), 350-355.

26. Poulton, R., Caspi, A., Milne, B. J., et al. (2002). Association between children's experience of socioeconomic disadvantage and adult health: A life-course study. Lancet, 360(9346), $1640-1645$

27. Costa, A., Mauritti, R., Martins, S., et al. (2002). Social classes in Europe. Portuguese Journal of Social Science, 1(1), 5-39.

28. Morotti, E., Battaglia, B., Paradisi, R., et al. (2013). Body mass index, stunkard figure rating scale, and sexuality in young italian women: A pilot study. The Journal of Sexual Medicine, 10(4), 1034-1043.

29. Benkeser, R. M., Biritwum, R., \& Hill, A. G. (2012). Prevalence of overweight and obesity and perception of healthy and desirable body size in urban, Ghanaian women. Ghana Medical Journal, 46(2), 66-75.

30. Gardner, R. M., Jappe, L. M., \& Gardner, L. (2009). Development and validation of a new figural drawing scale for bodyimage assessment: The BIAS-BD. Journal of Clinical Psychology, 65(1), 113-122.

31. Bulik, C. M., Wade, T. D., Heath, A. C., et al. (2001). Relating body mass index to figural stimuli: Population-based normative data for Caucasians. International Journal of Obesity and Related Metabolic Disorders, 25(10), 1517-1524.

32. Teixeira, C., Correia, S., Victora, C. G., et al. (2013). The Brazilian preference: Cesarean delivery among immigrants in Portugal. PLoS ONE, 8(3), e60168.

33. Howe, L. D., Galobardes, B., Matijasevich, A., et al. (2012). Measuring socio-economic position for epidemiological studies in low- and middle-income countries: A methods of measurement in epidemiology paper. International Journal of Epidemiology, 41(3), 871-886. 